\title{
Non-alcoholic fatty liver disease, bulb carotid intima-media thickness and obesity phenotypes: results of a prospective observational study
}

\author{
Anca D. Farcaş ${ }^{1}$, Camelia Larisa Vonica ${ }^{2}$, Adela C. Golea ${ }^{3}$ \\ ${ }^{1} 1^{\text {st }}$ Medical Clinic - Internal Medicine, Cardiology, and Gastroenterology, ${ }^{2}$ Diabetes, Nutrition, and Metabolic \\ Diseases Clinic, ${ }^{3}$ Emergency Medicine Department, "Iuliu Hatieganu" University of Medicine and Pharmacy, \\ Cluj-Napoca, Romania
}

\begin{abstract}
Aims: The objective of this prospective study was to assess the correlation between carotid intima-media thickness at the common carotid (CIMTc) and carotid bifurcation (CIMTb) level, hepatic fat accumulation, and obesity phenotypes. Material and methods: Two hundred obese adults, in which CIMTc and CIMTb thickness was determined, were included. According to body mass index (BMI) and presence of metabolic syndrome (MetS), patients were classified as metabolically healthy obese (MHO, obesity without MetS) and metabolically unhealthy obese (MUHO, obesity with MetS). MHO patients were further classified as MHO1 (obese with increased waist circumference) and MHO2 (obese with increased waist circumference plus one of the 4 criteria for MetS). Non-alcoholic fatty liver disease (NAFLD) presence was assessed by fatty liver index (FLI). Results: CIMTc and CIMTb increased with obesity phenotypes from $0.74 \mathrm{~mm}$ and $1.04 \mathrm{~mm}$ in $\mathrm{MHO} 1$ to $0.84 \mathrm{~mm}$ and 1.23 $\mathrm{mm}$ in $\mathrm{MHO} 2$ and $0.88 \mathrm{~mm}$ and $1.74 \mathrm{~mm}$ in MUHO. Obesity phenotypes were significantly correlated with CIMTb. NAFLD frequency increased from $66.0 \%$ in the MHO1 to $73.0 \%$ in the $\mathrm{MHO} 2$ and $84.2 \%$ in the MUHO (p<0.05). Independent of age, BMI, total cholesterol, HbA1c, and HOMA-IR, the CIMTc was significantly associated with FLI in all obesity phenotypes and CIMTb only in MHO2 and MUHO. Conclusions: Our results suggest that subclinical atherosclerosis varies according to obesity phenotypes and is correlated with the hepatic fat accumulation.
\end{abstract}

Keywords: carotid intima-media thickness; non-alcoholic fatty liver disease; obesity phenotypes

\section{Introduction}

Obesity represents a public health problem due to its increasing prevalence despite public awareness programs [1]. Based on cardiovascular (CV) risk factors, clinical studies have identified 2 types of obesity - metabolically healthy obesity (MHO) and metabolically unhealthy obesity (MUHO). MHO are characterized by the presence of obesity as defined by a body mass index (BMI) equal

Received 19.02.2017 Accepted 18.05.2017

Med Ultrason

2017, Vol. 19, No 3, 265-271

Corresponding author: Camelia Larisa Vonica

Medical Specialties Department - Diabetes,

Nutrition and Metabolic Diseases Clinic

8 Victor Babes Street

400012, Cluj-Napoca, Romania,

Phone: +40747642020, Fax: 0040264594455

E-mail: cami_umf@yahoo.com or over $30 \mathrm{~kg} / \mathrm{m}^{2}$ without metabolic $\mathrm{CV}$ risk factors. MUHO associates obesity with the presence of metabolic CV risk factors and an increased risk of diabetes and CV diseases [2-4]. Studies assessing the health risks associated with $\mathrm{MHO}$ have shown conflicting results, some showing similar or lower risk of $\mathrm{CV}$ disease and diabetes when compared to MUHO. Despite numerous clinical cross-sectional and prospective epidemiological studies evaluating the CV risk associated with this obesity phenotype and its clinical implications, controversies surrounding the health risks associated with MHO remain [5-7]. Therefore, it still under debate whether MHO represents a distinct phenotype compared with MUHO (lower health associated risks during lifetime or just a MUHO precursor) [8].

In parallel with the increasing prevalence of obesity an increased prevalence of nonalcoholic fatty liver disease (NAFLD) has been reported [9]. Obesity and ab- 
dominal obesity, metabolic syndrome, and type 2 diabetes have been identified among risk factors for NAFLD [10], which in turn is associated with an increased risk of fatal and non-fatal CV events and increased risk of total and CV diseases mortality [11]. The gold standard for NAFLD diagnosis is the liver biopsy but its invasive nature limits its use. Fatty liver index (FLI) score [12] is a noninvasive method widely used in epidemiological studies for NAFLD screening, showing good sensitivity compared with magnetic resonance spectroscopy for detecting fatty liver [13].

Carotid intima-media thickness (CIMT) is a simple and non-invasive method of the assessment of subclinical atherosclerosis and has been shown to be an independent predictor of CV disease risk [14-16]. Evaluation of CIMT includes evaluation of common carotid artery (CIMTc), bifurcation (bulb; CIMTb), and internal carotid artery and it has been shown that the association of the CIMT with CV risk factors varies according to the segment assessed [17]. The association between CIMT and NAFLD has been reported in the past years and this association was independent of other CV risk factors $[18,19]$.

Currently, limited data are available on the relationship between hepatic fat content and atherosclerosis according to obesity phenotypes. A recent study showed that MHO participants had significantly lower levels of CIMT and intrahepatic triglycerides content compared with the MUHO participants and intrahepatic triglycerides content was independently associated with metabolic syndrome (MetS) components and increased CIMT [20].

In this context, we aimed to investigate the correlation between subclinical carotid atherosclerosis assessed by CIMT at common carotid and carotid bifurcation level, hepatic fat, and obesity phenotypes.

\section{Material and methods}

This was a prospective study performed in the Emergency County Clinical Hospital Cluj-Napoca, Romania. We included 200 obese patients as defined by a BMI $\geq 30$ $\mathrm{kg} / \mathrm{m}^{2}$, who presented, between February 2014 and November 2015 for nutritional and metabolic status evaluation in the Diabetes, Nutrition and Metabolic Diseases Clinic. We excluded from the study patients under 18 years of age, with prior diagnosis of autoimmune, viral (hepatitis virus B, C, D), toxic or uncertain etiology hepatitis, with high alcohol consumption (>140 g/week), diabetes mellitus, pregnancy, with hypolipemic and/or weight loss medication. The patients signed an informed consent prior to enrollment and Institutional Ethics Committee approval was obtained.

\section{Clinical assessments}

We recorded for all patients demographic and clinical variables such age, weight, height, waist circumference, BMI [calculated as weight $(\mathrm{kg}) /$ height $\left(\mathrm{m}^{2}\right)$ ], associated disease (hypertension, dyslipidemia, etc), and their medication.

Waist circumference (WC) was measured with the patients standing, with a measuring tape halfway between the ribcage and the iliac crest, horizontally, at the end of a complete expiration.

Blood pressure (BP) was measured according to the guidelines [21] after a 5 minute resting, in sitting position. Two measurements were performed for each arm at 2 minutes interval. The arm with the highest BP was chosen and the average value of the measurements was computed.

\section{Metabolic syndrome definition}

Classifying patients as MHO or MUHO was performed after a series of explorations to identify the MetS components according to the International Diabetes Federation criteria [22]: 1) abdominal obesity: $\mathrm{WC}>94 \mathrm{~cm}$ (men) and $>80 \mathrm{~cm}$ (women); 2) hypertriglyceridemia: $\geq 150 \mathrm{mg} / \mathrm{dl}$; 3) low levels of HDL-C: $<40 \mathrm{mg} / \mathrm{dl}$ (men) and $<50 \mathrm{mg} / \mathrm{dl}$ (women) or specific treatment; 4) hypertension: $\geq 130 / 85 \mathrm{mmHg}$ or specific treatment; 5) high fasting glucose: $\geq 100 \mathrm{mg} / \mathrm{dl}$.

\section{Assay and indices assessment}

Blood samples were drawn from the cubital vein after a 12-h fasting. Fasting plasma glucose (FPG), triglycerides, total cholesterol, HDL-cholesterol, LDL-cholesterol, gamma-glutamyl transferase (GGT), transaminases, uric acid, fasting insulinemia (for insulinoresistance), glycated hemoglobin (HbA1c), apolipoprotein A1 and B were determined using a Beckman Coulter UniCel DxI 600. Insulin resistance was estimated using homeostasis model assessment (HOMA-IR) as [fasting glucose (mg/ $\mathrm{dL}) \times$ fasting insulin $(\mu \mathrm{UI} / \mathrm{mL})] / 405[23]$.

Based on FPG values, patients were classified as having dysglicemia (prediabetes) if they had an FPG level from 110 to less than $126 \mathrm{mg} / \mathrm{dl}$ [24]. NAFLD was diagnosed using the fatty liver index (FLI) score. FLI requires for calculation BMI, WC, triglycerides, and GGT [13]. A FLI score $>60$ is considered to be suggestive for the presence of NAFLD [13].

\section{Carotid Ultrasound Measurements}

Ultrasound evaluations were performed by a single examiner (with a 15-year expertise, certified for carotid ultrasonography) using a 3-10 MHz VF8-3 linear Transducer (ACUSON X300 Ultrasound System). The examination was performed with the patient lying in supine position, with a lateral probe position using a standardized protocol [25]. Wall thickness was measured in lon- 
gitudinal view, at the far wall level, with the transducer positioned strictly perpendicular to this wall (the lumenintima and media-adventitia interfaces were clearly defined), including the carotid bifurcation in the image plane. CIMT was measured at 10,15 , and $20 \mathrm{~mm}$ below the end of the common carotid artery (CCA), at a plaquefree point on the far wall and the average was considered the CIMTc on that side. Any atherosclerotic thickening $\geq 1.5 \mathrm{~mm}$ was considered a plaque. CIMTc was calculated as the average of the CIMT for left and right CCA. CIMTb was calculated as CIMT average in the thickest point (including plaque) of the left and right internal carotid bulb. Variation coefficients of the measurements for the examiner were $<5 \%$.

\section{Establishing the groups of patients according to obesity phenotype}

According to BMI and presence of MetS [22], participants were classified as $\mathrm{MHO}$ (obese without MetS) or MUHO (obese with MetS). MHO patients were further classified as MHO1 (obese with increased WC) and MHO2 (obese with increased WC plus one of the criterions for MetS).

\section{Statistical analysis}

Statistical analysis was performed with SPSS version 20. Kolmogorov-Smirnov tests were used to evaluate the distribution of investigated variables. Data was presented as proportions for qualitative variables, mean and standard deviation (SD) or median for continuous variables. The chi-square-test was used to compare categorical variables and t-test for continuous variables. Mann-Whitney test or Kruskal-Wallis test was used for non-normal distribution variables. Correlations were assessed with Spearman or Pearson coefficients, according to the variables distribution.

Univariate linear regression was performed for the relation between CIMT and FLI score in the whole sample and by obesity phenotype, multiple regression analysis was applied for the relation between CIMT and FLI adjusted for age, BMI, total cholesterol, $\mathrm{HbAlc}$, and HOMA-IR. A two-sided $p$ value $\leq 0.05$ was considered statistically significant.

\section{Results}

Baseline characteristics for the 200 patients who met the inclusion criteria and were included in the study are shown in Table I. MHO patients were younger, had significantly lower BMI, WC, systolic and diastolic BP comparing with MUHO. Comparing with $\mathrm{MHO}$, the MUHO patients had significantly lower HDL-cholesterol levels, higher triglycerides, uric acid, and CRP levels ( $p<0.05$ for all). The frequency of dysglycemia was $11.5 \%$ in the
MHO2 group and $74.0 \%$ in the MUHO group. In the MHO2 group 21 patients (40.4\%) had increased WC plus hypertension, 18 patients (34.6\%) plus low HDL-cholesterol, 7 patients $(13.5 \%)$ plus high triglycerides, and 6 patients (11.5\%) plus dysglycemia. CIMTc, CIMTb and FLI score were significantly higher in MUHO patients. .

Comparing $\mathrm{MHO} 1$ and $\mathrm{MHO} 2$ patients, those with MHO2 had significantly higher WC, glycemia, HbA1c, insulinemia, C-peptide, HOMA-IR, total and LDL-cholesterol, triglycerides, CRP and uric acid. CIMTc and CIMTb were significantly higher in $\mathrm{MHO} 2$ patients than in MHO1 patients. NAFLD frequency was $66.0 \%$ in the MHO1 and $73.0 \%$ in the MHO2 ( $<<0.05)$. FLI score increased from 63.44 in the MHO1 to 68.01 in the MHO2.

In all groups both CIMTc and CIMTb were directly and significantly correlated with FLI score. Correlation coefficients between CIMTc and FLI score were 0.343, 0.425 , and 0.343 (MHO1, MHO2, and MUHO, respectively); $0.763,0.443$, and 0.754 for CIMTb and FLI score in the three groups (Table II).

Obesity phenotypes were statistically significant correlated only with CIMTb ( $<<0.05$ for all; Table III).

In the univariate regression model, CIMTc was associated with the FLI score in the whole sample and CIMTb was associated with the FLI score only in the MHO1 group. After adjusting for age, BMI, total cholesterol, HbA1c, and HOMA-IR, a statistically significant association was observed between the CIMTb and FLI score in the MHO2 and MUHO, and CIMTc with the FLI score in all obesity phenotype groups (Table IV).

\section{Discussions}

MHO prevalence varies largely, up to $40 \%$, according to the clinical study and the definition criteria, being more common in younger persons $[8,26]$.

Our observations are similar to previous reports MHO has a lower CV risk profile compared to MUHO $[26,27]$. Elevated CIMTc and CIMTb, found in several MHO1 patients were correlated with ApoB/ApoA1 ratio, but not with LDL levels. Therefore, our results support the recommendations of the guidelines, to assess apoB even when LDL levels are normal because apoB correlates with atherosclerosis and predicts $\mathrm{CV}$ events [28].

Marini et al showed that CIMT increased from 0.68 $\mathrm{mm}$ in non-obese to 0.79 in $\mathrm{MHO}$ and 0.89 in obese with insulin resistance [27]. From our findings, increasing values were observed from $\mathrm{MHO} 1$ to $\mathrm{MHO}$, reaching the highest value in MUHO. Supposedly both CIMTc and CIMTb could increase with the number of MetS components. Previous studies have shown that CIMT and carotid plaque prevalence are associated with the 
Table I. Clinical, anthropometric, and metabolic characteristics of patients grouped according to the presence MetS.

\begin{tabular}{|c|c|c|c|c|c|c|}
\hline \multirow[t]{3}{*}{ Assessed parameters } & \multicolumn{4}{|l|}{ MHO } & \multirow{3}{*}{$\begin{array}{l}\text { MUHO } \\
\mathbf{N}=\mathbf{1 0 0}\end{array}$} & \multirow[t]{3}{*}{$\mathbf{p}^{\#}$} \\
\hline & All & MHO1 & MHO2 & $\mathbf{p}^{*}$ & & \\
\hline & $N=100$ & $\mathrm{~N}=48$ & $M=52$ & & & \\
\hline Age (years) & $41.02 \pm 11.33$ & $39.23 \pm 10.13$ & $42.33 \pm 12.12$ & 0.001 & $46.31 \pm 14.82$ & 0.001 \\
\hline Weight (kg) & $88.06 \pm 11.81$ & $88.06 \pm 11.81$ & $103.87 \pm 26.89$ & 0.001 & $103.87 \pm 26.89$ & 0.001 \\
\hline Waist $(\mathrm{cm})$ & $106.90 \pm 16.77$ & $100.00 \pm 10.27$ & $111.50 \pm 18.79$ & 0.01 & $118.47 \pm 12.51$ & 0.010 \\
\hline BMI $\left(\mathrm{kg} / \mathrm{m}^{2}\right)$ & $31.72 \pm 5.42$ & $29.98 \pm 4.45$ & $32.8 \pm 15.77$ & 0.23 & $36.40 \pm 5.75$ & 0.006 \\
\hline $\mathrm{SBP}(\mathrm{mmHg})$ & $127.13 \pm 22.64$ & $113.24 \pm 8.75$ & $134.21 \pm 10.13$ & 0.021 & $148.21 \pm 19.34$ & 0.002 \\
\hline DBP (mmHg) & $78.56 \pm 16.12$ & $62.44 \pm 6.21$ & $89.24 \pm 12.13$ & 0.024 & $95.21 \pm 19.43$ & 0.008 \\
\hline Total cholesterol (mg/dL) & $186.39 \pm 43.21$ & $179.60 \pm 43.37$ & $196.80 \pm 42.28$ & 0.023 & $206.63 \pm 50.23$ & 0.120 \\
\hline LDL-cholesterol (mg/dL) & $123.61 \pm 39.09$ & $105.14 \pm 24.81$ & $125.26 \pm 51.68$ & 0.015 & $130.26 \pm 51.68$ & 0.600 \\
\hline HDL-cholesterol (mg/dL) & $49.18 \pm 10.70$ & $52.13 \pm 8.02$ & $45.26 \pm 11.91$ & 0.01 & $41.20 \pm 11.84$ & 0.019 \\
\hline Triglycerides (mg/dL) & $106(83.23 ; 120.4)$ & $88(75.5 ; 111.5)$ & $112(91 ; 122.5)$ & 0.12 & $135(77 ; 191.5)$ & 0.002 \\
\hline ApoB/ApoA1 & $1.43 \pm 0.550$ & $0.85 \pm 0.33$ & $0.71 \pm 0.14$ & 0.23 & $1.44 \pm .230$ & 0.929 \\
\hline Glycemia (mg/dL) & $76.83 \pm 37.30$ & $72.75 \pm 26.94$ & $79.56 \pm 38.08$ & 0.01 & $97.28 \pm 36.13$ & 0.052 \\
\hline HbAlc (\%) & $5.55(5.3 ; 5.9)$ & $5.3(5.20 ; 5.45)$ & $5.6(5.45 ; 5.95)$ & 0.01 & $6.1(5.85 ; 6.25)$ & 0.001 \\
\hline Insulinemia $(\mu \mathrm{UI} / \mathrm{mL})$ & $9(7.5 ; 11.3)$ & $8.7(7.60 ; 9.30)$ & $10(8.15 ; 16.65)$ & 0.001 & $15.45(13.3 ; 23.85)$ & 0.027 \\
\hline C-peptide (ng/mL) & $2.25(2.1 ; 4.0)$ & $2.1(1.8 ; 2.2)$ & $2.7(2.2 ; 4.4)$ & 0.009 & $3.25(2.45 ; 5.65)$ & 0.038 \\
\hline HOMA-IR & $1.91(1.02 ; 5.45)$ & $2.43(1.02 ; 3.25)$ & $4.16(2.25 ; 6.22)$ & 0.001 & $6.12(5.44 ; 7.51)$ & 0.001 \\
\hline ASAT (IU/mL) & $23.02 \pm 11.45$ & $20.13 \pm 10.34$ & $26.47 \pm 14.03$ & 0.13 & $43.37 \pm 13.02$ & 0.035 \\
\hline ALAT (IU/mL) & $25.12 \pm 13.42$ & $21.03 \pm 11.02$ & $27.01 \pm 13.49$ & 0.19 & $45.23 \pm 15.27$ & 0.031 \\
\hline GGT (IU/mL) & $34.25 \pm 10.89$ & $24.38 \pm 11.02$ & $35.67 \pm 14.89$ & 0.21 & $48.38 \pm 16.72$ & 0.037 \\
\hline Uric acid (mg/dL) & $5.63 \pm 1.53$ & $4.96 \pm 1.20$ & $6.11 \pm 1.59$ & 0.001 & $6.70 \pm 1.05$ & 0.013 \\
\hline $\mathrm{CRP}(\mathrm{mg} / \mathrm{dL})$ & $1.90(1 ; 3.3)$ & $2.56(0.95 ; 3.10)$ & $1.40(1.05 ; 3.75)$ & 0.000 & $4.8(2.3 ; 7.5)$ & 0.035 \\
\hline CIMTc (mm) & $0.81 \pm 0.19$ & $0.74 \pm 0.17$ & $0.84 \pm 0.19$ & 0.001 & $0.88 \pm 0.17$ & 0.036 \\
\hline CIMTb (mm) & $1.2(1 ; 1.3)$ & $1.2(0.95 ; 1.3)$ & $1.2(1.1 ; 1.3)$ & 0.01 & $1.5(1.3 ; 2)$ & 0.008 \\
\hline FLI score & $66.65 \pm 26.52$ & $63.44 \pm 25.32$ & $68.01 \pm 26.82$ & 0.009 & $79.89 \pm 26.97$ & 0.002 \\
\hline NAFLD & 70.0 & 66.7 & 73 & 0.005 & 84.2 & 0.013 \\
\hline
\end{tabular}

The results are expressed as number $(\%)$, mean \pm SD or median $(\mathrm{Q} 1 ; \mathrm{Q} 3) .{ }^{*} \mathrm{p}$ values are provided for the comparison between MHO1 and MHO2.

${ }^{*} \mathrm{p}$ values are provided for the comparison between $\mathrm{MHO}$ and $\mathrm{MUHO} . \mathrm{N}=$ number of participants; $\mathrm{BMI}=$ body mass index; $\mathrm{SBP}=\mathrm{systolic}$ blood pressure; DBP = diastolic blood pressure; HbA1c - A1c glycated hemoglobin; HOMA-IR = homeostasis model assessment; ASAT $=$ aspartate transaminase; $\mathrm{ALAT}=$ alanine transaminase; $\mathrm{GGT}=$ gamma-glutamyl transferase $\mathrm{CRP}=\mathrm{C}$-reactive protein; CIMTc $=$ carotid intima-media thickness measured at common carotid artery level; CIMTb = carotid intima-media thickness measured at carotid bulb level; FLI = fat liver index; NAFLD = non-alcoholic fatty liver disease; $\mathrm{MHO}=$ metabolically healthy obese; MHO1 = obese with increased WC; $\mathrm{MHO} 2$ = obese with increased WC plus one of the criterions for MetS; MUHO = metabolically unhealthy obese.

presence of MetS [23, 29] and the number of its components [29-33]. Each additional component of the MetS is associated with a $0.02 \mathrm{~mm}$ increase in the CIMTc, independent of age, gender, family history of CVD, and smoking [34].

We found that only CIMTb was correlated with the obesity phenotype and systolic and diastolic BP in all groups, suggesting that BP-induced shear stress could explain the higher yearly growth rate of CIMTb compared to CIMTc [35]. Polak et al [17] showed that FPG and diastolic BP had a stronger association with CIMTc while hypertension, diabetes, and smoking with CIMTb.

We showed that FLI score increased in parallel with obesity phenotype groups. The prevalence of NAFLD increased from MHO1 to MUHO. Similarly, Zhang et al showed that the intrahepatic triglyceride content was significantly lower in MHO compared to MUHO and this content is a better predictor for MUHO than BMI, WC or percentage of body fat [20].

Intrahepatic fat accumulation and NAFLD are associated with a more adverse CV risk profile [36-40], while NAFLD is associated with insulin resistance, MetS and an atherogenic lipid profile [37, 41]. Furthermore, NAFLD patients have a higher prevalence of coronary artery lesions [36], higher CIMT, and atherosclerotic plaques [38], as well as a higher incidence of CVD, and increased CV mortality [39, 40]. We found significant correlations between the FLI score, presence of NAFLD, CIMTc, and CIMTb in all obesity phenotypes. Independent of age, BMI, total cholesterol, HbAlc, and HOMAIR, the CIMTc was significantly associated with FLI in all obesity phenotypes, unlike CIMTb (only in MHO2 and MUHO), suggesting that hepatic fat accumulation plays a role in the determination of the obesity phenotype asso- 
Table II. Correlations of carotid-intima media thickness with measured parameters according to the obesity phenotype.

\begin{tabular}{|c|c|c|c|c|c|c|}
\hline & MHO1 & & MHO2 & & MUHO & \\
\hline Assessed parameters & CIMTc & CIMTb & CIMTc & CIMTb & CIMTc & CIMTb \\
\hline Age (years) & $0.569 *$ & $0.523 *$ & 0.289 & 0.083 & $0.678 *$ & 0.550 \\
\hline Waist (cm) & 0.523 & 0.110 & $0.503 *$ & $0.336 *$ & -0.165 & 0.218 \\
\hline BMI (kg/m2) & 0.368 & 0.667 & 0.137 & -0.065 & -0.337 & -0.600 \\
\hline $\mathrm{SBP}(\mathrm{mmHg})$ & 0.505 & $0.514 *$ & 0.523 & $0.544 *$ & 0.505 & $0.603 *$ \\
\hline DBP (mmHg) & 0.489 & $0.563 *$ & 0.512 & $0.570 *$ & 0.489 & $0.563 *$ \\
\hline LDL cholesterol (mg/dL) & -0.413 & -0.306 & 0.001 & 0.355 & 0.297 & 0.218 \\
\hline HDL cholesterol (mg/dL) & 0.042 & 0.518 & 0.212 & -0.315 & 0.338 & 0.327 \\
\hline Triglycerides (mg/dL) & 0.200 & -0.409 & -0.056 & 0.222 & 0.463 & 0.491 \\
\hline ApoB/ApoA1 & $-0.629 *$ & -0.691 & -0.015 & 0.462 & -0.036 & -0.522 \\
\hline Glycemia (mg/dL) & $0.750 *$ & $0.710 *$ & 0.083 & -0.442 & -0.493 & 0.327 \\
\hline HbA1c (\%) & -0.209 & -0.662 & -0.110 & -0.050 & -0.021 & 0.806 \\
\hline HOMA-IR & -0.267 & -0.564 & 0.332 & -0.500 & 0.327 & 0.866 \\
\hline Uric acid (mg/dL) & 0.407 & 0.218 & 0.231 & 0.077 & 0.528 & 0.794 \\
\hline CRP (mg/dL) & -0.360 & 0.051 & 0.389 & $0.780 *$ & -0.118 & 0.500 \\
\hline FLI score\# & $0.343 *$ & $0.763 *$ & $0.425 *$ & $0.443 *$ & $0.343 *$ & $0.754 *$ \\
\hline NAFLD & $0.368 *$ & $0.792 *$ & $0.502 *$ & $0.434 *$ & $0.368 *$ & $0.783 *$ \\
\hline
\end{tabular}

$* \mathrm{p}$ values $<0.05$ showing a statistically significant association; \#FLI score included in the correlation analysis as a continuous variable; $\mathrm{N}=$ number of participants; $\mathrm{BMI}=$ body mass index; $\mathrm{SBP}=$ systolic blood pressure; $\mathrm{DBP}=$ diastolic blood pressure; HbA1c $-\mathrm{A} 1 \mathrm{c}$ glycated hemoglobin; HOMA-IR = homeostasis model assessment; $\mathrm{CRP}=\mathrm{C}$-reactive protein; CIMTc $=$ carotid intima-media thickness measured at common carotid artery level; $\mathrm{CIMTb}=$ carotid intima-media thickness measured at carotid bulb level; FLI = fat liver index; NAFLD = nonalcoholic fatty liver disease; $\mathrm{MHO}=$ metabolically healthy obese; $\mathrm{MHO} 1=$ obese with increased $\mathrm{WC}$; $\mathrm{MHO} 2=$ obese with increased WC plus one of the criterions for MetS; MUHO = metabolically unhealthy obese.

Table III. Correlations of the carotid-intima media thickness with the obesity phenotypes.

\begin{tabular}{|c|c|c|}
\hline Assessed parameters & MHO/MUHO & MHO1/MHO2/MUHO \\
\hline CIMTc & $0.286(\mathrm{p}=0.060)$ & $0.204(\mathrm{p}=0.184)$ \\
\hline CIMTb & $0.483(p=0.015)$ & $0.518(p=0.008)$ \\
\hline
\end{tabular}

CIMTc $=$ carotid intima-media thickness measured at common carotid artery level; CIMTb = carotid intima-media thickness measured at carotid bulb level; $\mathrm{MHO}=$ metabolically healthy obese; $\mathrm{MUHO}=$ metabolically unhealthy obese.

Table IV. Association of carotid-intima media thickness and with fatty liver index score in the whole sample and by obesity phenotypes

\begin{tabular}{lllll}
\hline Assessed parameters & CIMTc & & CIMTb & p \\
\cline { 2 - 5 } & $\boldsymbol{\beta}$ & $\mathbf{p}$ & $\boldsymbol{\beta}$ & 0.416 \\
\hline All sample & $\mathbf{0 . 2 8 3}$ & $\mathbf{0 . 0 0 5}$ & 0.072 & $<\mathbf{0 . 0 0 1}$ \\
MHO1 & 0.160 & 0.351 & 0.763 & 0.449 \\
MHO2 & 0.030 & 0.891 & 0.126 & 0.671 \\
MUHO & -0.151 & 0.378 & 0.052 & 0.763 \\
All sample\# & 0.116 & 0.226 & 0.058 & $-*$ \\
MHO1\# & $\mathbf{- 0 . 5 7 7}$ & $\mathbf{0 . 0 0 1}$ & $\mathbf{- *}$ & $\mathbf{0 . 0 0 1}$ \\
MHO2\# & $\mathbf{- 1 . 1 9 9}$ & $<\mathbf{0 . 0 0 1}$ & $\mathbf{1 . 5 8 6}$ & $\mathbf{0 . 0 0 4}$ \\
MUHO\# & $\mathbf{- 7 . 7 1 3}$ & $\mathbf{0 . 0 0 1}$ & $\mathbf{- 0 . 9 8 0}$ & \\
\hline
\end{tabular}

\#Models adjusted for age, BMI, total cholesterol, CRP, HbA1c, HOMA-IR. *Could not be calculated due to high number of covariates compared to sample size; $\beta$ - coefficient of correlation; CIMTc = carotid intima-media thickness measured at common carotid artery level; $\mathrm{CIMTb}=$ carotid intima-media thickness measured at carotid bulb level; $\mathrm{MHO}=$ metabolically healthy obese; $\mathrm{MHO} 1=$ obese with increased $\mathrm{WC} ; \mathrm{MHO} 2=$ obese with increased WC plus one of the criterions for MetS; MUHO = metabolically unhealthy obese.

ciated with subclinical atherosclerosis, probably through increased cytokines production [42]. Currently, there is scarce data on the association of CIMT and hepatic fat accumulation in obesity phenotypes. The only available study we could identify showed that irrespective of obesity phenotype and independent of percentage of body fat, an increase in the intrahepatic triglyceride content was associated with a higher risk of increased CIMT [20]. 
Our study has some limitations that we must acknowledge. The sample size was relatively small and therefore, future prospective evaluation in a larger scale study is required. We could not collect accurate information on the smoking status for all patients (years of smoking, passive smoking, etc) and therefore we could not assess its impact on CIMT. NAFLD was assessed by the FLI score and not by liver biopsy nor by an ultrasound exam. However, FLI score was shown to have a good performance in NAFLD identification $[43,44]$. The study subjects were selected from ambulatory patients and not from the general population. Also we did not take into account the different effects of associated therapy on MetS components (anti-inflammatory drugs, contraceptives, alternative therapies etc). Because of the small number of patients, dividing them into groups according to the association of MetS component would not have allowed an accurate statistical analysis. Therefore we do not have results on the impact of each MetS component - WC pair on NAFLD and CIMT.

\section{Conclusions}

Our results support previous findings suggesting the degree of subclinical atherosclerosis varies according to obesity phenotypes and is associated with hepatic fat accumulation. Hepatic fat accumulation increased according to the obesity phenotype and may represent a predictor of metabolic changes in obesity. Further studies investigating the association between NAFLD and CIMT progression in all obesity phenotypes are required.

\section{References}

1. Finucane MM, Stevens GA, Cowan MJ, et al; Global Burden of Metabolic Risk Factors of Chronic Diseases Collaborating Group (Body Mass Index). National, regional, and global trends in body-mass index since 1980: systematic analysis of health examination surveys and epidemiological studies with 960 country-years and 9.1 million participants. Lancet 2011;377:557-567.

2. Stefan N, Haring HU, Hu FB, Schulze MB. Metabolically healthy obesity: epidemiology, mechanisms, and clinical implications. Lancet Diabetes Endocrinol 2013;1:152-162.

3. Kim SH, Després JP, Koh KK. Obesity and cardiovascular disease: friend or foe? Eur Heart J 2016;37:3560-3568.

4. Mathew H, Farr OM, Mantzoros CS. Metabolic health and weight: Understanding metabolically unhealthy normal weight or metabolically healthy obese patients. Metabolism 2016;65:73-80.

5. Hinnouho GM, Czernichow S, Dugravot A, et al. Metabolically healthy obesity and the risk of cardiovascular disease and type 2 diabetes: the Whitehall II cohort study. Eur Heart J 2015;36:551-559.

6. Hamer M, Stamatakis E. Metabolically healthy obesity and risk of all-cause and cardiovascular disease mortality. J Clin Endocrinol Metab 2012;97:2482-2488.

7. Aung K, Lorenzo C, Hinojosa MA, Haffner SM. Risk of developing diabetes and cardiovascular disease in metabolically unhealthy normal-weight and metabolically healthy obese individuals. J Clin Endocrinol Metab 2014;99:462468.

8. Bala C, Craciun AE, Hancu N. Updating the Concept of Metabolically Healthy Obesity. Acta Endo (Buc) 2016;12:197205.

9. Welsh JA, Karpen S, Vos MB. Increasing prevalence of nonalcoholic fatty liver disease among United States adolescents, 1988-1994 to 2007-2010. J Pediatr 2013;162:496500.e1.

10. Lonardo A, Sookoian S, Chonchol M, Loria P, Targher G. Cardiovascular and systemic risk in nonalcoholic fatty liver disease - atherosclerosis as a major player in the natural course of NAFLD. Curr Pharm Des 2013;19:5177-5192.

11. Mikolasevic I, Milic S, Turk Wensveen T, et al. Nonalcoholic fatty liver disease - A multisystem disease? World J Gastroenterol 2016;22:9488-9505.

12. Bedogni G, Bellentani S, Miglioli L, et al. The Fatty Liver Index: a simple and accurate predictor of hepatic steatosis in the general population. BMC Gastroenterol 2006;6:33.

13. Cuthbertson DJ, O Weickert M, Lythgoe D, et al. External validation of the fatty liver index and lipid accumulation product indices, using $1 \mathrm{H}$-magnetic resonance spectroscopy, to identify hepatic steatosis in healthy controls and obese, insulin-resistant individuals. Eur J Endocrinol 2014; 171:561-569.

14. Chambless LE, Heiss G, Folsom AR, et al. Association of coronary heart disease incidence with carotid arterial wall thickness and major risk factors: the Atherosclerosis Risk in Communities (ARIC) Study, 1987-1993. Am J Epidemiol 1997; 146:483-494.

15. O’Leary DH, Polak JF, Kronmal RA, Manolio TA, Burke GL, Wolfson SK Jr. Carotid-artery intima and media thickness as a risk factor for myocardial infarction and stroke in older adults. Cardiovascular Health Study Collaborative Research Group. N Engl J Med 1999;340:14-22.

16. Polak JF, Pencina MJ, Pencina KM, O’Donnell CJ, Wolf PA, D'Agostino RB Sr. Carotid-wall intima-media thickness and cardiovascular events. N Engl J Med 2011;365:213221.

17. Polak JF, Person SD, Wei GS, et al. Segment-specific associations of carotid intima-media thickness with cardiovascular risk factors: the Coronary Artery Risk Development in Young Adults (CARDIA) study. Stroke 2010;41:9-15.

18. Cai J, Zhang S, Huang W. Association between nonalcoholic fatty liver disease and carotid atherosclerosis: a meta-analysis. Int J Clin Exp Med 2015;8:7673-7678.

19. Qu BG, Wang H, Liu YX, et al. Causes of Thickening of Carotid Intima-Media Thickness in Patients With Alcoholic 
Liver Disease: A Prospective Observational Study. Medicine (Baltimore) 2015;94:e1087.

20. Zhang H, Ma Z, Pan L, et al. Hepatic fat content is a determinant of metabolic phenotypes and increased carotid intima-media thickness in obese adults. Sci Rep 2016;6:21894.

21. Mancia G, Fagard R, Narkiewicz K, et al; Task Force for the Management of Arterial Hypertension of the European Society of Hypertension and the European Society of Cardiology. 2013 ESH/ESC Practice Guidelines for the Management of Arterial Hypertension. Blood Press 2014;23:3-16.

22. Alberti KG, Zimmet P, Shaw J; IDF Epidemiology Task Force Consensus Group. The metabolic syndrome--a new worldwide definition. Lancet 2005;366:1059-1062.

23. Matthews DR, Hosker JP, Rudenski AS, Naylor BA, Treacher DF, Turner RC. Homeostasis model assessment: insulin resistance and beta-cell function from fasting plasma glucose and insulin concentrations in man. Diabetologia 1985;28:412-419.

24. World Health Organization, International Diabetes Federation. Definition and diagnosis of diabetes mellitus and intermediate hyperglycemia: report of a WHO/IDF consultation. Geneva (CH): World Health Organization; 2006.

25. Touboul PJ, Hennerici MG, Meairs S, et al. Mannheim carotid intima-media thickness and plaque consensus (20042006-2011). An update on behalf of the advisory board of the $3 \mathrm{rd}, 4 \mathrm{th}$ and 5 th watching the risk symposia, at the 13th, 15th and 20th European Stroke Conferences, Mannheim, Germany, 2004, Brussels, Belgium, 2006, and Hamburg, Germany, 2011. Cerebrovasc Dis 2012;34:290-296

26. Phillips CM. Metabolically healthy obesity: definitions, determinants and clinical implications. Rev Endocr Metab Disord 2013;14:219-227.

27. Marini MA, Succurro E, Frontoni S, et al. Metabolically healthy but obese women have an intermediate cardiovascular risk profile between healthy nonobese women and obese insulin-resistant women. Diabetes Care 2007;30:2145-2147.

28. Lusis AJ. Atherosclerosis. Nature 2000;407:233-241

29. Pollex RL, Al-Shali KZ, House AA, et al. Relationship of the metabolic syndrome to carotid ultrasound traits. Cardiovasc Ultrasound 2006;4:28.

30. Rundek T, White H, Boden-Albala B, Jin Z, Elkind MS, Sacco RL. The metabolic syndrome and subclinical carotid atherosclerosis: the Northern Manhattan Study. J Cardiometab Syndr 2007;2:24-29.

31. Adolphe A, Cook LS, Huang X. A cross-sectional study of intima-media thickness, ethnicity, metabolic syndrome, and cardiovascular risk in 2268 study participants. Mayo Clin Proc 2009;84:221-228.

32. Fadini GP, Coracina A, Inchiostro S, Tiengo A, Avogaro A, De Kreutzenberg SV. A stepwise approach to assess the impact of clustering cardiometabolic risk factors on carotid intima-media thickness: the metabolic syndrome no-morethan-additive. Eur J Cardiovasc Prev Rehabil 2008;15:190196.

33. Ahluwalia N, Drouet L, Ruidavets J, et al. Metabolic syndrome is associated with markers of subclinical atherosclerosis in a French population-based sample. Atherosclerosis 2006; 186:345-353.

34. Panayiotou AG, Griffin M, Kouis P, et al. Association between presence of the metabolic syndrome and its components with carotid intima-media thickness and carotid and femoral plaque area: a population study. Diabetol Metab Syndr 2013;5:44.

35. Howard G, Sharrett AR, Heiss G, et al. Carotid artery intimal-medial thickness distribution in general populations as evaluated by B-mode ultrasound. ARIC Investigators. Stroke 1993;24:1297-1304.

36. Brea A, Puzo J. Non-alcoholic fatty liver disease and cardiovascular risk. Int J Cardiol 2013;167:1109-1117.

37. Assy N, Djibre A, Farah R, Grosovski M, Marmor A. Presence of coronary plaques in patients with nonalcoholic fatty liver disease. Radiology 2010;254:393-400.

38. Brea A, Mosquera D, Martin E, Arizti A, Cordero JL, Ros E. Nonalcoholic fatty liver disease is associated with carotid atherosclerosis: a case-control study. Arterioscler Thromb Vasc Biol 2005;25:1045-1050.

39. Matteoni CA, Younossi ZM, Gramlich T, Boparai N, Liu YC, Mc-Cullough AJ. Nonalcoholic fatty liver disease: a spectrum of clinical and pathological severity. Gastroenterology 1999;116:1413-1419.

40. Rafiq N, Bai C, Fang Y, et al. Long-term follow-up of patients with nonalcoholic fatty liver. Clin Gastroenterol Hepatol 2009; 7:234-238.

41. Silaghi CA, Silaghi H, Crăciun AE, et al. Age, abdominal obesity, and glycated hemoglobin are associated with carotid atherosclerosis in type 2 diabetes patients with nonalcoholic fatty liver disease. Med Ultrason 2015;17:300-307.

42. Shulman GI. Ectopic fat in insulin resistance, dyslipidemia, and cardiometabolic disease. N Engl J Med 2014;371:22372238.

43. Koehler EM, Schouten JN, Hansen BE, Hofman A, Stricker BH, Janssen HL. External validation of the fatty liver index for identifying nonalcoholic fatty liver disease in a population-based study. Clin Gastroenterol Hepatol 2013;11:1201-1204.

44. Carvalhana S, Leitão J, Alves AC, Bourbon M, Cortez-Pinto H. How good is controlled attenuation parameter and fatty liver index for assessing liver steatosis in general population: correlation with ultrasound. Liver Int 2014;34:e111-e117. 